\title{
„Bey solcher Gelegenheit treiben sie offt selber mit Christinnen Unzucht" Verbotene Sexualkontakte zwischen Juden und Christen in der Frühen Neuzeit
}

\begin{abstract}
"On such occasions they commit fornication with Christian women"
Illicit sexual intercourse between Jews and Christians in the Early Modern Period

Sexual intercourse between Jews and Christians was severely punished by the courts during the Middle Ages and the Early Modern Period. However, in many cases the allegations had been made up. The punishment was usually a high fine for the accused and not the death penalty mentioned in some legal texts. Evidence comes also from nonlegal sources, such as chronicles and other literary documents (e.g. the Meistersang). These sources do not refer to real incidents but voice unvarnished dislike or even outright hatred toward the Jews, instigated by religious fanaticism and greedy magistrates.
\end{abstract}

Keywords: early modern Europe - hatred of Jews - Jewish-Christian Relationship-Meistersang - sexual intercourse

\section{Forschungsstand}

Lange Zeit waren sexuelle Beziehungen zwischen Juden und Christen ein randständiges Thema der Forschung. Wenn überhaupt, dann interessierte sich die Rechtsgeschichte dafür, wobei eher die überlieferten Rechtsnormen als die meist nur aus Archiven zu rekonstruierende Rechtspraxis im Mittelpunkt standen. ${ }^{1}$ Dass aber gerade intime Kontakte zwischen Angehörigen beider Religionen ein Gradmesser für die Art des Zusammenlebens von Minderheit und Mehrheitsgesellschaft sein können, hat die Kontroverse um die These des jüdischen Historikers Cecil Roth (1899-1970) gezeigt. Dieser vertrat die Meinung, dass gerade das recht häufige

\footnotetext{
${ }^{1}$ Vgl. aus der älteren Literatur u. a. STOBвE, Juden 266f. Für neuere Arbeiten vgl. HÄRTER, Stellung der Juden 347-379, bes. 356f. KALLENBERG, Der Streit um den Judenpurschen.
}

Vorkommen von „süßen Sünden der Liebe“ (dolci peccati d'amore), also geschlechtliche Beziehungen von Juden und Christen in der Renaissance, darauf hindeute, dass Juden und Christen in der damaligen Zeit, zumindest in Italien, weitgehend harmonisch zusammengelebt hätten. ${ }^{2}$ Er stützt sich dabei auf ältere Forschungen zum spätmittelalterlichen Florenz. Danach sind allein in der zweiten Hälfte des 15. Jahrhunderts 34 Fälle von Sexualdelikten, an denen Juden beteiligt waren, in den Quellen überliefert. ${ }^{3}$ Roth deutet diese relativ hohe Zahl so, dass geschlechtliche Beziehungen zwischen Juden und Christen in dieser Zeit gleichsam an der Tagesordnung waren.

Inzwischen wissen wir, dass die Fälle, die gerichtsnotorisch wurden, keineswegs den Tatsa-

\footnotetext{
${ }^{2}$ RoTH, Gleanings, 210.

${ }^{3}$ CiARdini, I banchieri Ebrei. Vgl. TOAFF, Love 8.
} 
chen entsprechen müssen, denn mit solchen Beschuldigungen ließ sich leicht Geld verdienen, sowohl für den Informanten als auch für die städtische Obrigkeit, die immer wieder neue Ideen hatte, wie man von den Juden noch mehr Geld herauspressen konnte. ${ }^{4}$ Auch deuten Untersuchungen $\mathrm{zu}$ anderen italienischen Regionen in ungefähr demselben Zeitraum darauf hin, dass solche Delikte doch eher selten waren und weiterhin streng bestraft wurden, ${ }^{5}$ wenngleich die Todesstrafe im Spätmittelalter und in der Frühen Neuzeit - nicht allein in Italien - nur noch ausnahmsweise verhängt wurde. Insofern ist der Aussage der israelischen Historikerin Debra Kaplan zuzustimmen: "The sexual boundary was most closely guarded by both Jews and Christians, because symbolically, violation of that most intimate boundary was seen as a violation of communal religious identity." 6

Auch für andere europäische Städte und Länder liegen inzwischen Studien vor, in denen Sexualdelikte, an denen Juden beteiligt waren, quellenmäßig erfasst, allerdings nicht immer statistisch aufbereitet wurden. Für das spätmittelalterliche Spanien sind nicht wenige solcher „Unzuchtsfälle" überliefert.7 Doch trotz angedrohter Todesstrafe endeten alle Prozesse mit der Zahlung einer hohen Geldbuße durch den jüdischen „Täter". Zum Teil mussten die angeklagten Juden eine Geldstrafe zahlen, unabhängig davon, ob sie für schuldig befunden wurden oder nicht. Auch hier zeigt sich, dass bedingt durch fiskalische Interessen der weltlichen Obrigkeit ein falsches Bild von der Realität christlicherjüdischen Zusammenlebens entstehen konnte.

\footnotetext{
${ }^{4}$ BONFIL, Jews; CASSUTO, Gli ebrei a Firenze 229.

${ }^{5}$ Zur Lombardei vgl. BonfIL, Jews 105; zu Rom vgl. ADELMAN, Italian Jewish Women 156; zu Venedig vgl. Adelman, Servants 92; zu Modena vgl. ArON-Beller, Disciplining Jews 720.

${ }^{6}$ KaPlan, Because Our Wives Trade 258.

${ }^{7}$ Assis, Sexual behaviour 43.
}

Sex zwischen jüdischen Männern und ihren Sklavinnen scheint im Mittelmeerraum im späten Mittelalter gelegentlich ebenfalls vorgekommen bzw. aktenkundig geworden zu sein. ${ }^{8}$ In Spanien und Portugal, aber auch in Venedig achtete die Inquisition darauf, dass zum Christentum Zwangsbekehrte (conversos) sich nicht heimlich mit Jüdinnen einließen. Einige dieser Fälle wurden so gerichtskundig. ${ }^{9}$

Für Polen haben wir keine statistischen Angaben, vielmehr sind lediglich Einzelfälle überliefert, die nicht so recht zu dem düsteren Sittengemälde passen wollen, welches insbesondere der katholische Klerus zeichnete und sich auch als anti-jüdisches Stereotyp in der Gesetzgebung widerspiegelt. ${ }^{10}$

$\mathrm{Zu}$ Amsterdam liegen genauere Zahlen vor. Für den relativ kurzen Zeitraum 1600 bis 1623 lassen sich in den Quellen 13 Fälle von sexuellen Beziehungen zwischen portugiesischen Juden und Christinnen (meistens Dienstmägde) nachweisen. ${ }^{11}$

Mehrere neuere Studien existieren inzwischen zum Heiligen Römischen Reich Deutscher Nation. Darunter sind zwei, die genaue Zahlen über die Häufigkeit dieses Delikts liefern. Im 16. und 17. Jahrhundert kam es in Frankfurt am Main zu 71 Anklagen gegen Juden vor dem Ratsgericht, acht davon betreffen „Unzucht mit Christinnen", was einen Anteil von 11,3 Prozent an allen Bestrafungen von Angehörigen jüdischen Glaubens entspricht. ${ }^{12}$ Für das spätmittelalterliche Deutsche Reich konnte Annette BrunschwigSégal rund achtzig Fälle von Sexualbeziehungen zwischen Juden und Christen nachweisen.13 Lediglich einzelne Beispiele, aber dafür im Kontext, bringt Jörg R. Müller. Diese belegen, dass

\footnotetext{
8 SCHORSCH, Jews and Blacks 81.

${ }^{9}$ GiTLITZ, Secrecy 260-262.

${ }^{10} \mathrm{Vgl}$. KALIK, Fusion 67f.

${ }^{11}$ BODIAN, Hebrews 65.

12 KASPER-HOLTKOTte, Die jüdische Gemeinde.

${ }^{13}$ BRUNSCHWIG-SÉGAL, „der Jude habe si gemint“ 200.
} 
nicht nur unverheiratete jüdische Männer, sondern auch Ehemänner unter den Angeklagten waren. ${ }^{14}$

Die neuere Forschung lässt keinen Zweifel, dass sich trotz drakonischer, zumeist aber nur hoher Geldstrafen, die sicherlich auch abschreckend wirken sollten, Juden mit Christinnen einließen, manchmal auch umgekehrt. Selten waren es echte Liebesbeziehungen, die aufgrund des von beiden Religionen streng kontrollierten Mischehenverbots lediglich flüchtiger Art sein konnten, wenn sich nicht die Möglichkeit einer Konversion bot. Meist waren es ungleiche Sexualpartnerschaften auf Zeit (z. B. zwischen christlichen Prostituierten oder Dienstmägden und jüdischen Männern). Die Frage stellt sich, warum sich beide Seiten auf solche riskante Beziehungen einließen. Für Frauen aus der christlichen Unterschicht konnte Geld oder die Ausnutzung eines wirtschaftlichen Abhängigkeitsverhältnisses ein Grund gewesen sein, für die meist recht wohlhabenden jüdischen Männer wird man neben dem Stereotyp der Triebhaftigkeit andere Motive in Betracht ziehen müssen. Eine Erklärung könnte das sozialpsychologische Modell der Reaktanz liefern. Darunter versteht man eine Abwehrreaktion, die als Widerstand gegen äußere oder innere Einschränkungen gedeutet werden kann. Als Motivation kommt beispielsweise die massive Einschränkung von Freiheitsspielräumen durch Verbote in Frage. ${ }^{15} \mathrm{Zu}$ diesen als bedrückend empfundenen Repressionen zählte im Späten Mittelalter und in der Frühen Neuzeit vor allem das Verbot vor- und außerehelicher Beziehungen, auf dessen Einhaltung sowohl das Christentum als auch das Judentum achteten und im Falle der Zuwiderhandlung gegebenenfalls Sanktionen ergriffen. Als besonders strafwürdig galten Unzuchtsdelikte, wenn

\footnotetext{
${ }^{14}$ MÜLlER, Sexual Relationships 23, Anm. 16. Ein spektakulärer Einzelfall auch bei MüLLER, Sex and crime 395f.

${ }^{15}$ Miron, BREHM, Reactance Theory. 40 Years Later.
}

die Sexualpartner zudem noch unterschiedlichen Glaubens (Christen, Muslime, Juden) waren. Hier war der "Reiz des Verbotenen“ noch stärker, wenn sich die Gelegenheit bot, sich in face-to-face-Situationen näherzukommen.

Während wir über die Bestrafung solcher Beziehungen durch die christliche Obrigkeit zumeist gut unterrichtet sind, ist die innerjüdische Reaktion auf solche Verstöße einzelner Gemeindemitglieder weiterhin ein Forschungsdesiderat. ${ }^{16}$ Eine Reaktion konnte beispielsweise darin bestanden haben, die "Sünder" dem Spott der Gemeinde auszusetzen, indem man die Verfehlungen in einer Art jiddischem Bänkelsang einer breiten jüdischen Öffentlichkeit bekanntmachte. ${ }^{17}$

\section{Der jud mit Der Kristin}

In der Musikwissenschaft ist häufig darüber diskutiert worden, inwiefern sich Richard Wagners Antisemitismus auch in seiner Oper „Die Meistersinger" widerspiegelt. In diesem Zusammenhang wird zumeist auf die Figur des Beckmessers hingewiesen..$^{18}$ Bislang hat sich aber kaum jemand die Frage gestellt, ob sich nicht im frühneuzeitlichen deutschen Meistergesang zumindest Spuren eines Antijudaismus finden lassen. ${ }^{19}$ Angesichts der vielen Tausenden überlieferten Meisterlieder ist das kein einfaches Unterfangen. Aber es lohnt sich, wie das folgende, eher durch Zufall entdeckte Meisterlied aus Nürnberg zeigt:

\footnotetext{
${ }^{16}$ Vgl. TOAFF, Love 8, Anm. 7; Berger, Sexualität 93; WEINSTEIN, Marriage Rituals 226-228; WEINSTEIN, Juvenile sexuality 126; EPSTEIN, Sex Laws 172-178; BIALE, Eros and the Jews 142; STERN, Jewish identity $163 f$.

17 Vgl. ButZer, HÜtTENMEISTER, TREuE, Lied.

${ }^{18}$ Vgl. u. a. HeRTEL, Wagnerkult 124; BERGER, Beyond Reason 491.

${ }^{19}$ Nur kursorisch: HORTZITZ, Judenfeindschaft 38.
} 


\section{[-1-]}

„Höret ein selczame ge schicht! als man zelt Dreisig jare vnd finfzehen Hundert gar fein, Es sich zu drug zu Brag, das Bulen det ein jud ent wicht [= nichtsnutzig] Mit einer kristin zware [= freilich]. Das mocht nit lang ver borgen sein,Sunder es kam an tag.

Als ein Bidner Bichet $[=$ mit Pech $]$ ein fas,

Das jn wentig aus brente, welches gar Har er hiczet was,

Da muste er gar eilente

Sein manliches glit zu dem spund hin ein stecken, ver stet, zu einer straf, thue ich eüch kund; kein bit nit helfen det!

\section{[-2-]}

Als er vor schmercen schreien was, must er doch da beleiben, Bis das Bech Hart wur zu der zeit. auch liget man hie mit.

Ein schartets [= schartiges] messer auf das fas.

Der Schmerczen det ym dreiben,

Das er ab segen det be reit [= schon]

Sein manliches gelit.

Als er So plutig an dem ort

Dar von wolt laufen runde,

Hat man zu vor ver samlet dort

Ein haufen böser Hunde.

Die bisen yn gar Hart für war

E heim zu haus kam er.

Sein Bülerey ver ging ym gar mit der kristen weiber.

\section{[-3-]}

hie nem die Obri keit ein lehr, straf auch die vn zucht Eben, jedoch mit Be scheiden heit fein Beide an weib vnd man, Da mit sie nicht ein reiset sehr al hie in disem leben, auf das wir gott den herren rein auch nicht er zörnen than, vnd straf vns aucz mit vn gedult, wie oft ge schehen jste,

Das der, welicher Hat kein schult mit dem schultigen, wiste,

Die straf mus leiden hie auff ert.
Dar umb so fliehet fort

Die vn zucht, vnd eüch nit be schwert,

Gott straft sunst Hie vnd dort."

Der Verfasser nennt sich am Ende des Liedes, das am 30. Januar 1604 geschrieben wurde, selbst. Es ist ein gewisser Georg Hager, von Beruf - wie übrigens auch Hans Sachs - Schuhmacher. Gesungen werden sollte das Lied nach einer Melodie (Goldener Ton) von Walter von der Vogelweide (um 1170-um 1220).

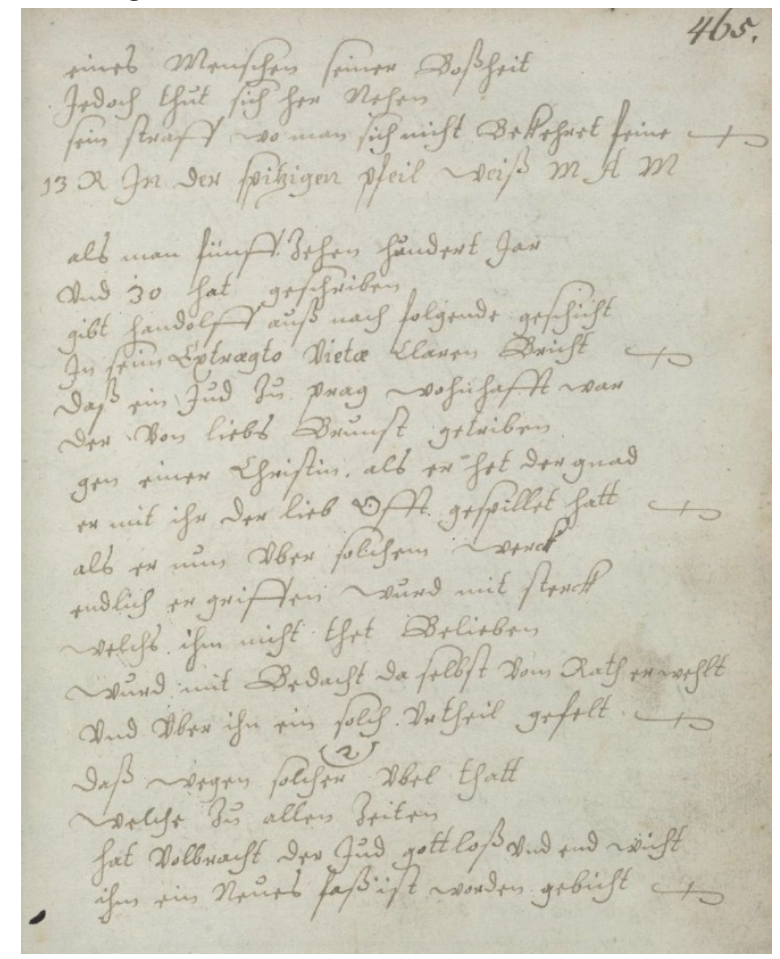

Abbildung 1: Georg Hagers Meistersingerlied von 1604 (Archiv der Stiftung Klassik Weimar Q 537).

Der Text ist uns in einer MeistersingerHandschrift, die sich heute in Wien befindet, überliefert. Es existiert eine moderne Transkription, aber kein Kommentar oder eine Analyse dieses merkwürdigen Textes, der offenbar ein historisches Ereignis anspielt, um daraus eine Moral zu ziehen. ${ }^{20}$

Worum geht es? Der Kern der Geschichte, die wir bereits gehört haben, ist rasch nacherzählt. Angeblich wurde ein Jude 1530 in Prag grausam

\footnotetext{
${ }^{20}$ Bell, Hager, Nr. 588. Vgl. BrunNer, WACHINGER, Repertorium 7, 437.
} 
bestraft, weil er Unzucht mit einer Christin begangen hatte. Er wurde dazu verurteilt, seinen Penis in das Spundloch eines Fasses zu stecken, das mit heißem Pech gefüllt war. Als die Schmerzen unerträglich wurden, schnitt er mit einem Messer, das man ihm absichtlich auf das Fass gelegt hatte, sein Glied ab. Als er sich dann blutend auf dem Weg nach Hause machte, verfolgte ihn eine Meute Hunde, die man zusammengetrieben hatte.

Bevor wir uns auf der Suche nach der Quelle dieser Kriminalgeschichte machen, sei noch etwas zum Verfasser gesagt. ${ }^{21}$ Dieser war, wie bereits erwähnt, von Beruf Schuhmacher, stammt also aus dem Handwerkermilieu. Er wurde 1552 in Nürnberg als Sohn des gleichnamigen Georg Hager, der Ältere (1512-1571), der übrigens bei Hans Sachs das Schuhmacherhandwerk erlernt hatte, geboren. Er war in den 1570er-Jahren einige Jahre auf Wanderschaft, unter anderem in Breslau, wo er in der Werkstatt Wolff Herolds, einem bekannten Meistersinger, arbeitete. Seinen ersten Unterricht im Meistersang erhielt er doch schon viel früher durch seinen Vater. Seit 1587 wirkte er regelmäBig an der Nürnberger Singschule mit und übernahm 1608 sogar das ehrenvolle Amt des "Merkers". Er besaß am Lebensende eine 13 Bände umfassende Sammlung von Meisterliedern, die er teilweise selbst geschrieben, teilweise zusammengetragen hatte. Bestattet wurde er 1634 auf dem Rochusfriedhof in Nürnberg.

Das hier interessierende Meisterlied stammt nachweislich aus eigener Feder. Hager, das muss man für die Interpretation wissen, dichtete im selben Jahr noch zwei weitere Texte zum Thema „Unzucht" mit den bezeichnenden Titeln „wie der ehebruch ge strafft wirt" und „Die vol Bewrin mit jrmen knecht" ${ }^{22}$ Ausweislich der Notizen des Nürnberger Scharfrichter Meister

\footnotetext{
${ }^{21}$ STAHL, Meistersinger 179-184.

${ }^{22}$ BeLL, Hager II, Nr. 977 vom 27.1.1604, IV, Nr. 1206 vom 21. 2. 1604.
}

Franz gab es in dieser Zeit keine spektakulären Fälle von Unzucht, die an Leib und Leben gestraft wurden. ${ }^{23}$ Hintergrund könnte eher eine Häufung von solchen Vorkommnissen in der Nachbarschaft oder im Bekanntenkreis Hagers gewesen sein, die eventuell mit Kirchenstrafen und Geldbußen geahndet wurden. Wie die „Moral der Geschichte" belegt, geht es dem Verfasser weniger darum, die verbotenen Beziehungen zwischen einem Juden und einer Christin zu brandmarken, als an diesem Beispiel zu zeigen, wie die Obrigkeit Unzucht je nach Schwere mit Augenmaß strafen soll. Ein antijüdischer Unterton lässt sich jedenfalls in diesem Meisterlied nicht entdecken.

\section{Ein anderes Lied, dieselbe Geschichte}

Vierzehn Jahre später erzählte ein anderer Nürnberger Meistersinger dieselbe Geschichte, doch nun mit deutlich antijüdischer Stoßrichtung. Von diesem Lied existiert bis heute keine Transkription. ${ }^{24}$ Der Text lautet in meiner Übertragung:

„als man fünff zehen hundert Jar vnd 30 hat geschriben gibt handolff auß nach folgende geschicht In sein Extragto Vietae Claren Bricht $\omega$ daß ein Jud zu prag wohnhafft war der von liebs Brunst getriben gen einer Christin, als er het der gnad er mit ihr der lieb offt gespillet hatt $\omega$ als er nun vber solchen werck endlich ergriffen wurde mit sterck [= Gewalt] welchs ihn nicht thet Belieben wurd mit Bedacht da selbst vom Rath erwehlt Vnd Vber ihn ein solch Vrtheil gefelt $\omega$

\footnotetext{
${ }^{23}$ Tagebuch des Meister Franz Scharfrichter. Zu dieser Quelle vgl. HARRINGTOn, Ehre des Scharfrichters. ${ }^{24}$ Archiv der Klassik Stiftung Weimar Q537, fol. $465 \mathrm{r} / \mathrm{v}$.
} 
[-2-]

Das wegen solcher Vbel thatt

Welche zu allen Zeiten

Hat Volbracht der Jud gottloß vnd end wicht [= nichtsnutzig]

Ihm ein Neues faß ist worden gebicht $\emptyset$

In welches er on alle gnad

von Mäniglich [= in mancher Hinsicht]

vnbscheiden [ $=$ unkorrekt]

weil das selbig noch Brennen thet Jetzund

sein Manlichs glid stecken must in deß spund $\emptyset$ auch wurd ihm gelegt auff das faß

ein stumpffentes Messer fürbaß

groß pein der Jud thet leiden

als er solches leiden kund nicht mehr

dan ihm die hitz des faß thet Brenen sehr 0

Er dises Messer Nam ver rucht [= ruchlos]

Vnd thet ohn alles verdrissen

Ihm abschneiden sein Manlichs glid mit qual

Darauß daß Blutt thet flissen ohne Zahl $\emptyset$

Vnd zu Sal führen [= salvieren] in die flucht

er sich hoch hatt Beflissen

als den selben ersehen thet Jetzund

des Volckes Meng so vmb die Refür [= Bezirk]

stund $\omega$

hetzten sie hund an ihn mit schmach

welche ihn da zu grimer Rach

ellend haben zerryssen

auß dissen man kann lehrnen Jeder Zeit

wie man sich hütten sol für vn keuschheit $\oplus$

1628 den 25 Marti d: wolff winter"

Bei dem jung verstorbenen Autor Wolff Winter (1611-1632) handelt es sich um den Bruder des Nürnberger Messerschmieds Hans Winter (1591-1627), der einige hundert Meisterlieder gedichtet hat. ${ }^{25}$ Auch Wolff Winter war in diesem Handwerk tätig. In einer Singschule trat er erstmals 1626 auf. Ansonsten erfahren wir noch aus einem Trauermeisterlied seines Freundes Ambrosius Metzger (1573-1632) vom 20. Juli

${ }^{25}$ STAHL, Meistersinger 326f.
1632, dass der Verstorbene das Theaterspielen („Agirn“) liebte und viel komponierte. ${ }^{26}$

Der Inhalt seines Meisterlieds differiert in mehrfacher Hinsicht von dem uns bekannten Text Georg Hagers dem Jüngeren. So findet hier der wegen Unzucht mit einer Christin bestrafte Jude ein jämmerliches Ende, indem er von einer Hundemeute in den Tod gehetzt wird. Wenngleich die Lehre, die der Hörer bzw. Leser aus dieser Geschichte ziehen soll, ähnlich ist, nämlich sich vor Unzucht zu hüten, so hat dieses Meisterlied zugleich auch eine antijüdische Spitze, wie aus der Wortwahl hervorgeht (,,von liebs Brunst getriben", "gottloß", „zu grimer Rach“). Bedeutsam an diesem Meisterlied ist aber noch etwas ganz Anderes, dass nämlich gleich zu Anfang die historische Quelle, der die Geschichte entnommen wurde, genannt ist. Es handelt sich um das Werk von Andreas Hondorf (15301572) mit dem Titel „Promptvarium exemplorum“ (1572). Dort wird unter den Exempeln, die die Einhaltung des neunten und zehnten Gebotes einschärfen sollen, folgende Begebenheit geschildert:

„Anno Christ 1530. Ist eine Jude zu Praga in Behemen gewesen / so mit einer Christin gebulet / vnnd drüber ergriffen / da hat er müssen sein Mennlich Glied zu einem Spunde eines gepichten brennenden Fasses hinein stecken vnd wurd jm darzu auffs Faß ein schartig stumpff messer geleget / als jhm nun die hitze / so grimmig wehe gethan / hat jhm mit dem Messer sein Gliedt vor schmertz abgeschnitten / Vnndt da er nun also blutig hat daruon lauffen wöllen / hat man böse hunde an jhn gehetzet / die jhn zurissen haben. ${ }^{27}$

\footnotetext{
${ }^{26}$ Meisterlied 2Met/843. Nachweis bei BRUNNER, WACHINGER, Repertorium Bd. 8, 462. Vgl. STAHL, Meistersinger 328.

${ }^{27}$ HONDORFF, Promptvarium exemplorum (1572) 350(r). In der ersten Auflage von 1568 ist diese Geschichte noch nicht kolportiert.
} 
Über Andreas Hondorf, der Pfarrer im sächsischen Droyßig war, ist kaum etwas bekannt, außer dass er zwei umfangreiche theologische Werke verfasst und zum Druck gebracht hat. ${ }^{28}$ Sein populärer (protestantischer!) Heiligenkalender, der bis 1610 zehn Auflagen erlebte, kam allerdings erst ein Jahr nach seinem Tod heraus und wurde von seinem Mitarbeiter Vincentius Sturm fortgesetzt. ${ }^{29}$ Schaut man sich die Quelle genauer an, so erkennt man, dass Hondorf aus einem anderen Werk die Informationen über den Prager Vorfall im Jahr 1530 entnommen hat. Es handelt sich um eine Art Universallexikon der Frühen Neuzeit, das erstmals 1565 in Basel von Theodor Zwinger dem Älteren (1533-1588) publiziert wurde. ${ }^{30}$ Hondorf hat nachweislich die erweiterte Auflage von 1571 benutzt. Die Vorlage ist in Latein und lautet wie folgt:

"Pragae in Bohemia anno Christi 1530. Iudaeus, qui cum Christiana rem habuerat, dolio picto per foramen virile pudendum indere iussus, serrato \& obtuso cultro iuxtà apposito, cum vi caloris torquerentur, sibi jpsi partes quas peccauerat amputavit: cumq[ue] cruentus fugeret, immissis atrocissimis canibus laceratus est." 31

Diese Geschichte wird nicht nur von Hondorf schon sehr früh übernommen, sondern findet sich auch in frühneuzeitlichen Bibelkommentaren sowie in aus unterschiedlichen Quellen kompilierten Chroniken. ${ }^{32}$

Wie heute bei Wikipedia, so muss man auch bei Enzyklopädien des 16. Jahrhunderts eine gewisse Skepsis an den Tag legen, was den Wahrheitsgehalt betrifft. Nachforschungen in Prager Archiven brachten bislang keinen Hinweis auf eine so spektakuläre Bestrafung eines Juden.

\footnotetext{
${ }^{28}$ DUNKEL, Historisch-Critische Nachrichten 217.

${ }^{29}$ POHLIG, Zwischen Gelehrsamkeit 451.

${ }^{30}$ ZEDELMAIER, Navigieren im Text-Universum 113.

31 ZWINGER, Theatrum vitae humanae.

${ }^{32}$ Vgl. z.B. BÜTTNER, Epitome historiarum 337; LORIN, Commentarij 957; BEYERLINCK, Magnum theatrum 107.
}

Allerdings ist die Quellenüberlieferung für die Prager Strafgerichtsbarkeit in diesem Zeitraum rudimentär. Von Kennern der böhmischen Kriminalitätsgeschichte wird diese Geschichte aber als wenig glaubhaft eingeschätzt. Für diese Meinung sprechen mindestens zwei wesentliche Gründe: Wie Karel Malý gezeigt hat, waren sexuelle Beziehungen zwischen Juden und Christen im böhmischen Recht des 15. bis 16. Jahrhunderts nicht strafbar (d.h. über Rahmen des Deliktes der Unzucht bzw. des Ehebruches hinaus). ${ }^{33}$ Der einzige, aber letzendlich nicht erfolgreiche Versuch, speziell den Tatbestand des Geschlechtsverkehrs zwischen Juden und Christen ins böhmische Recht aufzunehmen, stellt das Rechtsbuch „Práva městská“ (Stadtrechte) aus dem Jahr 1536 dar. Verfasser war der Prager Bürger und Jurist Brikcí von Licsko (1488-1544). ${ }^{34}$ Darin ist auch ein Teil der Judendekrete von Papst Clemens VI. (um 1290-1352) übernommen worden. Brikcís Rechtsbuch war aber kein geltendes Recht. ${ }^{35}$

\footnotetext{
${ }^{33}$ MALÝ, Trestní právo 245.

34 SLAVÍČKOVÁ, Guardianship 108.

${ }^{35}$ Für diese Hinweise danke ich PhDr. Petr Kreuz vom Stadtarchiv Prag.
} 


\section{Eine weitere Variante: Die Frankfurter Schuhmacherchronik von 1611}

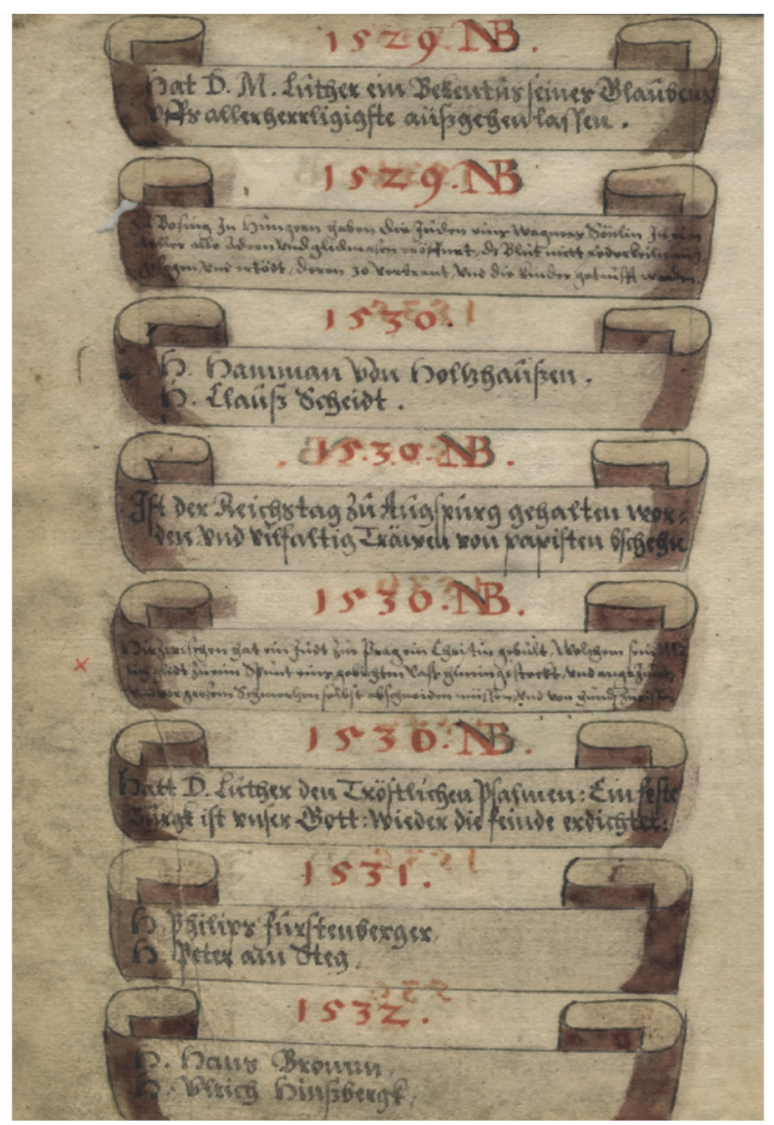

Abbildung 2: Frankfurter Bürgermeisterliste für die Jahre 1529 bis 1532 (Institut für Stadtgeschichte Frankfurt, Handwerker Bücher 117, fol. 18v).

1611 verfasste der Frankfurter Schuhmacher Balthasar Wolffstern (1576-1631) eine Chronik seines Handwerks, die auch Ämterlisten enthält, darunter eine Aufzählung der Personen, die in der Stadt am Main im 15., 16. und frühen 17. Jahrhundert das Bürgermeisteramt ausübten. ${ }^{36} \mathrm{Zu}$ den jeweiligen Jahren des Amtsantritts finden sich verschiedene Nota Bene-Vermerke, die auf andere wichtige Ereignisse in dem betreffenden Jahr verweisen. Zu 1530 wird notiert: „Diezwischen hat ein Judt zue Prag ein Christin gebult, welchem sein Mänlich glidt zu eim Spunt eines gebichten vaße hineingesteckt

\footnotetext{
36 SCHMIDT, Traditionen 299 u. 311.
}

vnd angezundt, vnd vor grosem schmertzen selbst abschneiden müssen vnd von hund zerissen. “ ${ }^{\prime 37}$

Die Quelle wird nicht genannt, aber vermutlich dürfte Wolffstern Zwingers Enzyklopädie oder ein vergleichbares Werk gekannt haben. Es fällt auf, dass es nicht die einzige judenfeindliche Bemerkung des Autors ist, der sich als glühender Verehrer Luthers durch seine häufigen diesbezüglichen Einträge zu erkennen gibt. Erwähnt werden unter anderem eine angebliche Hostienschändung in Franken im Jahr 1298 (fol. 10v), ein Judenpogrom in Nördlingen 1384 (fol.11r), eine angebliche Brunnenvergiftung durch Juden in Straßburg 1349 (fol. 11v), eine Ritualmordbeschuldigung in Triest 1476 (fol. 14r), ein weiteres solches Ereignis im Friaul 1479 (fol. 14r), eine Hostienschändung und eine Judenverbrennung in Brandenburg 1510 (fol. 16v) sowie ein weiterer Ritualmordvorwurf in Bösing (Pezinok) in Ungarn im Jahr 1529 (fol. 18v). Ab 1560 finden sich dann keine weitere antijüdischen Bemerkungen mehr in dieser Bürgermeisterliste. Wir wissen, dass der letzte Eintrag aus dem Jahr 1626 stammt. ${ }^{38}$ Warum Wolffstern neben den bekannten, in der antijüdischen Literatur immer wieder angeführten Judenpogromen wegen angeblicher Hostienschändung, Ritualmord und Brunnenvergiftung auch die seltsame und weniger bekannte Prager Episode berichtet, lässt sich nur vermuten. Hintergrund könnte Fälle von sexuellen Beziehungen zwischen Juden und Christinnen sein, die gegen Ende des 16. und zu Beginn des 17. Jahrhunderts sicherlich Stadtgespräch in Frankfurt waren. Ein jiddisches Lied, das um 1600 entstanden sein muss, prangert einen Juden mit Namen Leib zu Roseneck an, der in der Stadt am Main (hinter dem Holzzaun) eine (vermutlich christliche)

\footnotetext{
${ }^{37}$ Institut für Stadtgeschichte, Frankfurt: Handwerker, Bücher 117, fol. 18v.

${ }^{38}$ SCHMIDT, Traditionen 229.
} 
Magd geschwängert haben soll. ${ }^{39}$ Aktenkundig ist ein weiterer Fall: 1609 wurde der verheiratete Jude Amschel zu Rad verhaftet, weil er mit mehreren Dirnen geschlechtlich verkehrt haben soll. Er wurde inhaftiert, mit der Folter bedroht und schließlich dazu verurteilt, die Stadt nie wieder zu betreten. Auch seine Familie wurde ausgewiesen. Eine der christlichen Prostituierten, mit der er sich eingelassen hatte, wurde, obwohl sie hochschwanger war, ebenfalls der Stadt verwiesen, mit der Androhung, sie bei einer Rückkehr zu ertränken. ${ }^{40}$ Eine andere Dirne setzte man im selben Jahr wegen desgleichen Delikts über den Main in einen Korb oder auf einen Balken, dann wurde sie zur Belustigung des Publikums in das Wasser "geschnellt“. ${ }^{41}$

\section{Motivgeschichte}

Wenn es sich bei der Bestrafung eines Juden, die sich 1530 in Prag zugetragen haben soll, um eine fiktive Geschichte handelt, dann muss diese dennoch glaubwürdig klingen. ${ }^{42}$ Wie wir noch sehen werden, ist die Kastration eines Juden für ein (Sexual-)Verbrechen im Mittelalter in einigen wenigen Fällen bezeugt. Aber solche historischen „Vorbilder" dürften bei der Konstruktion dieser aufsehenerregenden Kriminalgeschichte eher keine oder nur eine geringe Rolle gespielt haben.

Dass ein Jude so leichtsinnig sein kann, es zu riskieren, dass sein Penis abgeschnitten wird, ist zum Beispiel das Thema einer frühneuzeitlichen Papstbiographie, die bereits die Forschung zu Shakespeares Kaufmann von Venedig interes-

\footnotetext{
39 ButZer, HÜTtEnMEISTER, TREue, Lied 30.

${ }^{40}$ ANDERNACHT, Regesten zur Geschichte der Juden Nr. 3816, 3818, 3822, 3824-3826.

${ }^{41}$ Ebd., Nr. 3820.

${ }^{42}$ Allgemein zur Glaubwürdigkeit von Kriminalquellen: ZEMON DAVIS, Fiction in the Archives.
}

siert hat. ${ }^{43}$ Danach soll Papst Sixtus V. (15211590) im Fall einer Wette entschieden haben, in dem es darum ging, dass ein Jude namens Sampson Seneda mit einem christlichen Kaufmann über den Wahrheitsgehalt einer Nachricht gewettet hatte und die Einlösung der Wette darin bestand, dem Verlierer ein Pfund Fleisch von einem Teil des Körper abzuschneiden, den man aus Sittsamkeit besser nicht nennen möchte (e che la modestia non vuo che io nome ${ }^{44}$ ). Ähnliche zeitgenössische Sexual- oder Bestrafungsphantasien dürften bei der Ausschmückung des fiktiven Prager Unzuchtfalls eine Rolle gespielt haben, denn es ist ja der Jude, der sich, um die furchtbaren Schmerzen loszuwerden, entmannt, also die Kastration sich selbst zuzuschreiben hat. Weiterhin ist zu beachten, dass es keine Vorbilder in der damaligen Strafpraxis dafür gibt, dass jemand zur Strafe sein Glied in den Spund eines mit heißem Pech gefüllten Fasses stecken musste. Aber es lassen sich dennoch Bezüge zum mittelalterlichen Strafrecht nachweisen, die den Verfasser inspiriert haben könnten. $\mathrm{Zu}$ den grausamen Strafverschärfungen, die bei der Exekution von Juden gelegentlich zur Anwendung kamen, gehört, dass man dem jüdischen Delinquenten eine mit brennendem Pech gefüllte Holzschale auf das Haupt setzte, damit man ihn von einem gehängten Christen unterscheiden konnte. So sah es jedenfalls das spätmittelalterlich Stadtrecht von Brünn vor. ${ }^{45}$ Darauf spielt auch das folgende jiddische Sprichwort an: "Oyfn ganef brent dos hitl“ ( (היטל), Auf einem Dieb brennt der Hut". ${ }^{46}$ Dass diese Norm durchaus gelegentlich der Strafpraxis entsprach, zeigt der folgende Fall: 1463 hängte man in Nürnberg einen auswärtigen Juden

\footnotetext{
${ }^{43}$ Vgl. u.a. SHAPIRO, Shakespeare and the Jews 122 124.

${ }^{44}$ LETI, Vita di Sisto Quinto pontefice romano 84.

${ }^{45}$ RÖSSLER, Stadtrechte von Brünn 202. Vgl. dazu auch GLANZ, Jewish 3-26, bes. 7.

${ }^{46}$ Silverman-WeinReich, Yiddish Proverb 67.
} 
wegen Verrats. Ihm wurde zur Strafverschärfung eine Haube mit heißem Pech auf den Kopf aufgesetzt, so „das im das pech über die augen floß, und man hieng keinen hunt zu im“. ${ }^{47}$

Das hier bereits erwähnte Hunde-Motiv wird seit langem in der jüdischen Rechtsgeschichte diskutiert. Im Schweizer Kanton Glarus sollten jüdische Diebe an einem eigenen Galgen aufgehängt werden, und zwar an den Füßen zwischen zwei wütenden und beißenden Hunden. ${ }^{48}$ Aber zwischen Rechtsvorschrift und Rechtspraxis liegen oft Welten, wie die moderne Kriminalitätsgeschichte gezeigt hat. Gleichwohl finden sich in den Quellen vereinzelt Fälle, wo Hunde Juden bei der Hinrichtung tatsächlich am Galgen an den Pfoten aufgehängt zur Seite gegeben wurden. ${ }^{49}$ Dass man Hunde als Tiere wählte, war kein Zufall, denn Juden wurden von Christen seit der Spätantike als „Hunde“ bezeichnet, die es auf den Leib Christi in Gestalt von Hostien abgesehen hatten. ${ }^{50}$

Bei der Motivsuche muss man jedoch weniger an konkrete Rechtsfälle oder Strafbestimmungen als Vorbilder denken, sondern vor allem an den Einfluss, den die spätmittelalterliche und frühneuzeitlichen Schwankliteratur, aber auch Novellen gehabt haben könnten. Darin kommt nämlich das Motiv des entdeckten und des mit Entmannung bestraften Ehebruchs recht häufig vor. Meist handelt es sich um Priester, die wegen ihrer sexuellen Eskapaden mit einer untreuen Frau kastriert werden. ${ }^{51}$ „Das Handlungsschema gibt vor", so die Mediävistin Susan Tuchel, „daß Pfaffen von untreuen Ehefrauen

\footnotetext{
${ }^{47}$ Historische Commission, Chroniken der fränkischen Städte 285. Vgl. MüLLER, Beziehungsnetze 81, Anm. 30; SCHUSTER, Verbrecher 178 (mit falscher Jahreszahl).

${ }^{48}$ GLANZ, Execution 6f.

${ }^{49}$ MÜller, Beziehungsnetze 80-82, Anm. 30 u. 31.

${ }^{50}$ STOW, Jewish Dogs XIV-XV; FREY, „Wölt Gott man hing sie wie die Hund“.

${ }^{51}$ Vgl. Burrows, Stereotype 166-199; KurzOVÀ, Priests in Dyer's Vat.
}

als die besseren Liebhaber bevorzugt werden; deren verbotene und nimmersatte Lust bot sich als Ausgangspunkt für Vorstellungen und Phantasien von entmännlichenden Strafen an [...]." ${ }^{\prime 52}$ Speziell als Vorlage könnte in unserem Zusammenhang die Geschichte gedient haben, die der Florentiner Autor Franco Sacchetti (um 13321400) in seiner Novellensammlung Trecentonovelle erzählt. In der 25. Novelle wird geschildert, wie Dolcibene im Auftrag des Magistrates der Stadt Forlì einen wegen Unzuchts verurteilten Priester vor einer großen Menschenmenge wie folgt bestraft: Dolcibene kriecht in ein Fass, auf das sich der Verurteilte rittlings setzen muss. Dessen Geschlechtsteile stecken im Spundloch, so dass er dem Priester mit einem Rasiermesser ohne großen Aufwand die Hoden abschneiden kann und diese dann in Beutel steckt und mitnimmt. Der auf diese Weise grausam bestrafte Priester muss sich daraufhin mehrere Tage von einem Arzt behandeln lassen und versucht anschließend, dem Scharfrichter die inzwischen getrockneten und gepökelten Testikel durch einen Mittelsmann gegen einen hohen Betrag abzukaufen, „denn der kastrierte Priester könne ohne sie nicht die Messe lesen." ${ }^{53}$ Wie dieses Beispiel zeigt, zeichnen sich die Novellen im Unterschied zu den Schwänken durch eine besondere Drastik und Realistik in Hinblick auf den Akt der Kastration aus. Nach Tuchel scheint es, dass sich dieses Motiv im Laufe des Mittelalters zwar nicht grundsätzlich verändert hat, aber „sich die Kastrationsgeschichten aus ihrem traditionellen Rahmen zumeist pfäffischer Ehebruchshandlungen lösen und in andere Kontexte treten." ${ }^{45}$ Dafür könnte auch die angeblich wahre Geschichte aus Prag ein Beispiel sein, indem hier der lüsterne Pfaffe gegen den geilen

\footnotetext{
52 TUCHEL, Kastration 260.

53 SACCHETTI, Novellen Bd. 1, 94. Vgl. Beutin Aggression 50-51, EsCH, Wahre Geschichten 14f.; TUCHEL, Kastration 279.

${ }^{54}$ TUCHEL, Kastration 283.
} 
Juden - auch ein altes Stereotyp - ausgetauscht wird. Der einzige Unterschied würde lediglich darin bestehen, dass die Bestrafung am sündhaften Glied in diesem Fall tödlich endet.

Zum Umfeld dieses Motivkomplexes gehört auch die aus dem Mittelalter überlieferte Vision, dass man aufgrund eines unstatthaften sexuellen Begehrens aus dem Traum aufwacht und feststellt, dass das sündhafte Glied in einem verwitterten Baumstamm steckt (uirilem ipsius uirgam in quodam foramine factu). ${ }^{55}$

\section{Kastration als Strafe für Juden}

In der Rechtsgeschichte wird die Kastration als sogenannte Talionsstrafe bezeichnet. Es wird also das Körperglied bestraft, das von der Tat betroffen beziehungsweise an dieser beteiligt war. ${ }^{56}$ Oft beruft man sich dabei fälschlicherweise auf den alttestamentarischen Rechtsgrundsatzes עין תמחת עין aus dem Buch Exodus, wo es heißt: „[...] so sollst du geben Leben für Leben, Auge für Auge, Zahn für Zahn, Hand für Hand, Fuß für Fuß, Brandmal für Brandmal, Wunde für Wunde, Strieme für Strieme" (2. Mose 21:23-25). Damit war vor allem ein angemessener Schadensersatz bei Körperverletzungsdelikten gemeint, um Blutrache zu verhindern, wie in der rabbinischen Auslegung immer wieder betont wird. Dieses jüdische Rechtsprinzip wurde später in christlichen Texten als "Auge um Auge, Zahn um Zahn“ übersetzt und damit als Vergeltungsstrafe gedeutet. Letztere Rechtsauffassung findet auch Ausdruck in der Strafe der Entmannung, die vor allem bei Sittlichkeitsverbrechen zur Anwendung kam.

\footnotetext{
${ }^{55}$ EASTING, SHARP, Peter of Cornwall's Book of Revelations 136, Zeilen 9-10. Vgl. auch FIRTH GREEN, Elf Queens 182f. Ich danke Peter Dinzelbacher für den Hinweis.

${ }^{56}$ Zum männlichen Glied als Symbol der Sexualität im christlich-jüdischen Kontext vgl. STEINBERG, The Sexuality of Christ 383.
}

Dafür findet sich in der Rechtgeschichte eine Fülle von Belegen, sowohl im Geltungsbereich des römischen als auch des germanischen Rechts. ${ }^{57}$

Das Römische Recht sah für Juden die Kastration als Strafe nicht vor, da diese bereits durch die Beschneidung sich einer Art der Selbstentmannung unterzogen hatten. ${ }^{58}$ Erst im deutschen Recht des Spätmittelalters findet sich eine solche Strafbestimmung: Ein Mainzer Weistum von 1422 bestimmt:

„Auch wan ein waltpode einen juden bei einer christenfrauwen oder maide funde, unkeischeit mit ir zu triben, die mag er beide halten. do sol man dem juden sein ding abesniden $u$. ein aug usstechen, und sie mit ruden us jagen, oder sie mogen umb eine summe darumb dingen. ${ }^{459}$

Dass diese Strafe jemals im Mainzer Herrschaftsgebiet zur Anwendung gekommen ist, darf mit Fug und Recht bezweifelt werden. Es existieren jedenfalls keine weiteren Quellenbelege. Außerdem lässt das Weistum bereits die Möglichkeit zu, durch eine Geldbuße die Sache zu erledigen. Auch im Stadtrecht von Macerata aus dem Jahre 1553 ist eine solche Strafe vorgesehen, wenn ein Jude mit einer Christin sexuell verkehrt. Der Passus lautet im Original: "Iudaeus vero qui christianam cognoscere carnaliter ausus fuerit, puniatur poena mortis naturalis, prius genitalibus \& lingua excisis, cum publicatione bonorum." 60 Auf diesem Delikt stand also nicht nur die Todesstrafe, zudem sollten dem Delinquenten vor der Hinrichtung das Geschlechtsteil und die Zunge abgeschnitten werden.

\footnotetext{
${ }^{57}$ Vgl. GÜNTHER, Wiedervergeltung 261; BROWE, Entmannung 74-76; VAN EICKELS, Gendered Violence; FINUCCI, The Manly Masquerade 249.

58 Bullough, BRundage, Medieval Sexuality 84.

${ }^{59}$ GRIMM, Weisthümer 533. Vgl. GMELIN, Abhandlung 60f.; SCHAAB, Diplomatische Geschichte 104; STOBвE, Juden 152 u. Anm. 151.

${ }^{60}$ Volumen statutorum civitatis Maceratae. [Macerata] 1553, 38. Vgl. LA MANTIA, Roma 444.
} 
Dass es gelegentlich im Mittelalter tatsächlich zu einer Kastration eines Juden wegen eines Sexualdeliktes gekommen ist, lässt sich gleichwohl nicht abstreiten. Allerdings sind solche Fälle ganz selten überliefert. 1308 war der berühmte italienische Jurist Oldradus de Ponte (?-1335) in Avignon Zeuge, wie ein Jude wegen sexueller Beziehungen zu einer Christin entmannt wurde. In seinem Consilium Nr. 333, das von der Bestrafung solcher Delikte handelt, ${ }^{61}$ heißt es am Ende:

"Sed Dominus R. de Apulea, et Dominus Ant. de Cruce, qui tunc preerant in officio iudicatus Avinion, dictum Iudeum in amissione virilium condemnaverunt, cuius nomen erat Pandonus, et ego vidi virilia incisa ante palatium." 62

Der Rechtsgelehrte, der sich in seinem Rechtsgutachten gegen eine solche strenge Bestrafung (abscissium genitalium) aussprach, da es sich nicht um einen Fall von Ehebruch, sondern um einen Geschlechtsverkehr mit einer Prostituierten aus reiner "Fleischeslust" handelte, berichtet also, dass er mit ansah, wie einem Juden mit Namen Pandonus vor dem Palast zur Strafe die Geschlechtsteile abgeschnitten wurden. ${ }^{63}$

1341 wurde in dem südfranzösischen Städtchen Manosque ein jüdischer Arzt mit Namen Crescas von Mirabeau angeklagt, der Mutter einer Patientin sexuelle Avancen gemacht zu haben. Er wurde auf frischer Tat ertappt und vom Gericht zur Kastration verurteilt. Es bestehen aber Zweifel daran, ob die Strafe wirklich ausgeführt wurde oder ob es dem Verurteilten nicht doch gelang - wie so oft im Rechtalltag - durch eine hohe Geldbuße den Verlust seiner Männlichkeit

\footnotetext{
${ }^{61}$ VAlseCCHI, Oldrado da Ponte 675 Anm. 20; MAZZANTI, Habere rem cum iudea 5

${ }^{62}$ Oldradus DE PONTE, Consilia, questiones \& allegatione, consilium CCCXXXXIII. Vgl. auch die moderne Edition von ZACOUR, Jews and Saracens 90f. (Latein), 68f. (engl. Übersetzung).

${ }^{63}$ Vgl. SCHNITZLER, Juden vor Gericht 297f.
}

abzuwenden. ${ }^{64} 1378$ drohte dasselbe Schicksal einem gewissen Joseph Sireyson in Châteauneuf-de-Gadagne, einer kleinen Gemeinde im heutigen Département Vaucluse in Südfrankreich gelegen. Auch er konnte sich vermutlich dieser Strafe durch eine Appellation an ein höheres Gericht entziehen. ${ }^{65}$

Ein weiterer Fall findet sich im Tagebuch des päpstlichen Zeremonienmeisters Johannes Burckard (manchmal auch Burchard oder Burckardus genannt, um 1450-1506) aus den Jahren 1483 bis 1506. Dort wird unter anderem ein Ereignis beschrieben, dass sich 1498 zugetragen haben soll. Anfang April dieses Jahres hatte man eine Kurtisane namens Cursetta ins Gefängnis geworfen, weil sie sich mit einem Mauren eingelassen hatte, der Frauenkleider trug. Beide wurden zur Strafe zusammen durch die Stadt geführt, sie in einem von oben bis unten offenen, gürtellosen schwarzen Samtkleid, ihr Liebhaber in seinem Frauengewand, das bis zum Nabel aufgeschürzt war, so dass man seine Genitalien sehen und damit seine falsche Identität erkennen konnte. Nach dem Umzug durch die Straßen ließ man Cursetta frei, der Maure kam wieder ins Gefängnis. Am 7. April 1498 führte man ihn zusammen mit zwei Räubern zur Hinrichtungsstätte. Ein Gerichtsdiener, der einen Stock in der Hand hielt, an dessen Spitze die beiden Hoden angebunden waren, die man zuvor einem Juden herausgeschnitten hatte, weil er mit einer Christin Unzucht begangen hatte, ritt auf einem Esel voran. Die Delinquenten wurden auf das Flora-Feld gebracht, wo die beiden Räuber auf die übliche Weise gehängt wurden, den Mauren dagegen band man mit einem Strick um den Hals an einen Pfahl inmitten eines Holzstoßes, nachdem man ihn vorher getötet hatte. Dann wurde der Scheiterhaufen angezündet, doch weil es stark zu regnen be-

\footnotetext{
${ }^{64}$ Vgl. ShatZMilleR, Jews 89f.; Rodrigue Lavoie, La délinquance sexuelle 379 .

${ }^{65}$ BARDINET, La condition civile 35.
} 
gann, wollte das Feuer nicht recht brennen, so dass nur die Schenkel des Mannes verkohlten. ${ }^{66}$ Über das Schicksal des Juden, den man zuvor entmannt hatte und dessen Geschlechtsteile während der Hinrichtung eines Mauren zur Schau gestellt wurden, erfahren wir aus dieser Quelle leider nichts.

Letztmals ist die Kastration eines Juden wegen eines Sexualdelikts für das Jahr 1569 in einer Quelle aus Mantua belegt. Allerdings wurde ein solches Urteil nicht verkündet, sondern lediglich als eine Bestrafungsmöglichkeit vom Gericht in Erwägung gezogen. ${ }^{67}$

Wie sehr die Kastration als Strafe für Juden selbst im 17. Jahrhundert noch eine Option zumindest in den Augen der Bevölkerung war, belegt ein französischer Text aus dem Bestand der Mazarinaden. ${ }^{68}$ Einer Verbrecherbande, die angeblich aus Juden bestand und einen jungen Pariser Bürger mit Namen Jean Bourgeois auf der Straße ermordet haben soll, wurde von dem anonymen Verfasser dieser Flugschrift bereits im Untertitel die Kastration angedroht. ${ }^{69}$ Und noch hundert Jahre später verlangte ein anonymes antijüdisches Pamphlet aus England, dass Juden für bestimmte Straftaten - darunter auch sexueller Verkehr mit Christinnen - mit Kastration bestraft werden sollten. ${ }^{70}$

$\mathrm{Ob}$ sich hinter solchen Bestrafungsforderungen und Strafphantasien auch eine Art Kastrationsangst $^{71}$ verbirgt, wie sie Sigmund Freud (18561939) für die ödipale Phase der Kindheit beschrieben hat, mag dahin gestellt bleiben. Wenn

\footnotetext{
${ }^{66}$ BURCHARDI, Diarium 2, 443f.; dt. Übersetzung bei GEIGER, Alexander VI. und sein Hof 190f. Vgl. RIEGER, Juden in Rom 2, 443f; HIRSCHFELD, Homosexualität 206.

${ }^{67}$ SIMONSOHN, History of the Jews 115, Anm. 49.

${ }^{68}$ Vgl. LÉVI, L'Affaire Bourgeois; FABRE-Vassas, Singular Beast 115.

${ }^{69}$ Jugement criminel].

${ }^{70}$ [SWIFT, HoweLL], An Historical and law-treatise 32. Vgl. dazu FelsensteIN, Anti-Semitic Stereotypes 69.

${ }^{71}$ FREUD, Hemmung 137.
}

überhaupt, dann müsste man in Anlehnung an den französischen Psychoanalytiker Jacques Lacan (1901-1981) eine solche Kastration, die angedroht bleibt, eher als „symbolische Kastration “72 bezeichnen. In diesem Zusammenhang spielt nicht zuletzt das antisemitische Stereotyp eine Rolle, dass die Beschneidung bereits eine Art unvollendeter Kastration sei.73 Als Beleg mag die Stelle in einer berüchtigten Hetzschrift des frühen 19. Jahrhunderts dienen. Darin wird gefordert: „Damit die Juden sich ferner nicht fortpflanzten, könnte man auch Alles, was von ihnen an die Wand $\mathrm{p}-\mathrm{k}-\mathrm{lt}$, statt es beschneiden, künftig verschneiden lassen. ${ }^{\text {“74 }}$

\section{Literarisches Nachleben}

Bis heute hat man in der rechtsgeschichtlichen Literatur den Wahrheitsgehalt des merkwürdigen Falls der Selbstkastration eines der Unzucht mit einer Christin beschuldigten Prager Juden nie in Zweifel gezogen. Vor allem in älteren Abhandlungen zur Geschichte des Judenrechts ist eine solch harte Bestrafung im Laufe der Jahrhunderte immer wieder zitiert oder kolportiert worden. ${ }^{75}$ Die juristischen Texte enthalten sich dabei aber zumeist jeder Wertung. Anders dagegen die Werke mit eindeutig antijüdischer Tendenz, wie z. B. Johann Jacob Schudts (1664-1722) „Jüdische Merckwürdigkei-

\footnotetext{
${ }^{72}$ LACAN, Bedeutung des Phallus 129. Vgl. TAYLOR, Castration.

${ }^{73}$ Vgl. u.a. BOYARIN, Unheroic Conduct 326; FABREVASSAS, Singular Beast 117.

${ }^{74}$ HUNDT-RADOWSKY, Judenspiegel 108.

${ }^{75}$ Eine Auswahl in zeitlicher Reihenfolge: BONIFACIO, Historia Ludicra 9; WUCHERFEIND, Der verdammliche Juden-Spieß, 209f.; DÖPLER, Theatrum poenarum, I, 1022-1023 und II, 429; WAGENSEIL, Locvlamentvm Tertivm 134; FRANCKENSTEIN, Dinglinger, Diss. jur. de iuribus singularibus circa Judaeos 47-48.; WILDVOGEL, Lochner, Dodecas legalis 38; BECK, Tractatus de juribus Judaeorum $364 f$.
} 
ten" $(1714 / 16)^{76}$, die in der Frühen Neuzeit und zum Teil auch darüber hinaus breit rezipiert wurden. Ähnliches gilt für das Werk des Tiroler Arztes und Sittenpredigers Hippolytus Guarinonius (1571-1654). ${ }^{77} \mathrm{Im}$ 19. Jahrhundert erwähnt der österreichische Jurist Joseph Freiherr von Hormayr (1781 oder 1782-1848), der sich über Kollegen gelegentlich antisemitisch äußerte, ${ }^{78}$ dass man den "fleischlichen Umgang zwischen Juden und Christen [...] früher grausamer bestraft" habe und verweist dabei auf den uns bekannten Fall, allerdings mit falscher Jahreszahl (1548) und falschem Ort (Ungarn). ${ }^{79}$ Dagegen wusste der nationalsozialistische Autor, der 1937 die grausame Bestrafung eines Juden zu Prag im Jahr 1530 mit Häme schildert, diese durchaus historisch präzise einzuordnen. ${ }^{80} \mathrm{Nach}$ dem Ende des Nationalsozialismus geriet diese angeblich wahre Geschichte, die wir als eine Art frühneuzeitliche „urban legend“81 oder Großstadtsage entlarven konnten, in Vergessenheit. Jedenfalls findet sich so gut wie keine Erwähnung mehr, weder in der rechtsgeschichtlichen Literatur noch in Werken zur jüdischen Geschichte, mit einer Ausnahme: in einem Buch über Antisemitismus und Sexualität von $2008 .{ }^{82}$

\footnotetext{
${ }^{76}$ SCHUDT, Jüdische Merckwürdigkeiten IV, VI. Buch, 18. Kap., 334f.

77 GUARINONIUS, Grewel der Verwüstung 1130.

78 VORDERMAYER, Antisemitismus 227.

${ }^{79}$ HORMAYR, Anemonen 1350.

${ }^{80}$ RUMBERG, Der außereheliche geschlechtliche Umgang 399.

${ }^{81}$ Zum Begriff und zu Beispielen moderner Großstadtmythen vgl. BRUNVAND, Vanishing Hitchhiker; HARDER, Lexikon der Großstadtmythen; KANESHIROHAUPTMANN, Das ist absolut wahr!

${ }^{82}$ HensCHel, Neidgeschrei.
}

\section{Korrespondenz:}

Hon.-Prof. Dr. Dr. h. c. Robert JÜTTE

Institut für Geschichte der Medizin

der Robert Bosch Stiftung

Straußweg 17

70184 Stuttgart

robert.juette@igm-bosch.de

ORCID-Nr. 0000-0002-5266-3936

\section{Abkürzungen:}

Siehe das allgemeine Abkürzungsverzeichnis: [http://www.rechtsgeschichte.at/files/abk.pdf]

\section{Literatur:}

Howard Zvi ADELMAN, Italian Jewish Women, in: Judith R. BASKIN (Hg.), Jewish Women in Historical Perspective (Detroit 21991) 150-168.

DERS., Servants and Sexuality. Seduction, Surrogacy, and Rape. Some Observations concerning Class, Gender and Race in Early-Modern Italian Jewish Families, in: T. M. RUDAVSKY (Hg.), Gender and Judaism (New York 1995) 81-97.

Dietrich ANDERNACHT, Regesten zur Geschichte der Juden in der Reichsstadt Frankfurt am Main von 1520-1616, Teil 2 (Hannover 2007).

Katherine ARON-BELLER, Disciplining Jews. The Papal Inquisition of Modena 1598-1630, in: The Sixteenth Century Journal 41 (2010) 713-729.

Yom Tov Assis, Sexual behaviour in mediaeval Hispano-Jewish society, in: Ada RAPOPORT-ALBERT, Steven ZIPPERSTEIN (Hgg.), Jewish History. Essays in Honour of Chimen Abramsky (London 1988) 25-59.

Léon BARDINET, La condition civile des Juifs du Comtat Venaissin pendant le séjour des papes (1309-1376), in: Revue historique 12 (1880) 1-47.

Johann Jodocus BECK, Tractatus de juribus Judaeorum, von recht der Juden. [...] (Nürnberg 1731).

Karol Berger, Beyond Reason. Wagner Contra Nietzsche (Oakland 2017).

Ruth BERGER, Sexualität, Ehe und Familienleben in der jüdischen Moralliteratur 900-1900 (Wiesbaden 2003).

Wolfgang BEUTIN, Aggression als Kastration in Erzähltexten der Renaissance, in: Freiburger literaturpsychologische Gespräche 6 (Würzburg 1987) 43-63. 
Laurentius BEYERLINCK, Magnum theatrum vitae humanae: hoc est, rerum diuinarum, humanarumque syntagma catholicum, philosophicum, historicum, et dogmaticum. Ad normam Polyantheae vniuersalis dispositum. [...]. Bd. 2 (Lyon 1665).

David BIALE, Eros and the Jews. From Biblical Israel to Contemporary America (New York 1992).

Miriam BodiAN, Hebrews of the Portuguese Nation. Conversos and Community in Early Modern Amsterdam (Bloomington 1997).

Robert BonfIL, Jews, Christians, and sex in Renaissance Italy. A historiographical problem, in: Jewish History 26 (2012) 101-111.

Baldassare BONIFACIO, Historia Ludicra. Opus Ex Omni Disciplinarum Genere, Selecta \& Jucunda Eruditione Refertum (Bruxellae 1656).

Peter BROWE, Zur Geschichte der Entmannung. Eine religions- und rechtsgeschichtliche Studie (Breslau 1936).

Horst BRUNNER, Burghart WACHINGER (Hgg.), Repertorium der Sangsprüche und Meisterlieder des 12. bis 18. Jahrhunderts, 16 Bde. (Tübingen 19862002).

Jan Harold BRUNVAND, The Vanishing Hitchhiker: American Urban Legends \& Their Meanings (New York 1981).

Annette BRUNSCHWIG-SÉGAL, „der Jude habe si gemint". Verbotene Beziehungen zwischen Juden und Christen im Spätmittelalter, in: Judaica 57 (2001) 182-203.

Johannis BURCHARDI, Diarium, sive rerum urbanarum commentarii 3 Bde. (Paris 1883-1885).

Vern L. Bullough, James A. Brundage (Hgg.), Handbook of Medieval Sexuality (New York 1996).

Darron BURROWS, The stereotype of the priest in the Old French fabliaux (Bern 2005).

Evi Butzer, Nathanja HütTEnMEISTER, Wolfgang TREUE, „Ich will euch sagen von einem bösen Stück ...". Ein jiddisches Lied über sexuelle Vergehen und deren Bestrafung aus dem frühen 17. Jahrhundert, in: Aschkenas, Zeitschrift für Geschichte und Kultur der Juden 15 (2005) 25-53.

Wolfgang BÜTTNER, Epitome historiarum christlicher ausgelesener Historien und Geschichten [...] (o. O. 1576).

Umberto CAssuto, Gli ebrei a Firenze nell'età del Rinascimento (Firenze 1918).

Marino CIARDINI, I banchieri Ebrei in Firenze nel secolo XV e il Monte di Pietà fondato da Girolamo Savonarola. Appunti di storia economica con appendice di documenti (Borgo San Lorenzo 1907).
Jacob DÖPLER, Theatrum poenarum, suppliciorum et executionum criminalium oder Schau-Platz [...] 2 Teile (Sonderhausen 1693, 1697).

Johann Gottlob Wilhelm DUNKEL, Historisch-Critische Nachrichten von verstorbenen Gelehrten und deren Schriften, Bd. 3, Ausgabe 1 (Köthen-Dessau 1757)

Richard FIRTH GREEN, Elf Queens and Holy Friars. Fairy Beliefs and the Medieval Church (Philadelphia 2016).

Robert EASTING, Richard SHARP (Hgg.), Peter of Cornwall's Book of Revelations (Toronto 2013).

Louis M. EpsteIN, Sex Laws and Customs in Judaism (New York 1948).

Arnold EscH, Wahre Geschichten aus dem Mittelalter. Kleine Schicksale selbst erzählt in Schreiben an den Papst (München 2010).

Claudine FABRE-VASSAS, The Singular Beast. Jews, Christians \& the Pig (New York 1997).

Frank FELSENSTEIN, Anti-Semitic Stereotypes. A Paradigm of Otherness in English Popular Culture 1660-1830 (Baltimore 1995).

Valeria FINUCCI, The Manly Masquerade. Masculinity, Paternity, and Castration in the Italian Renaissance (Durham-London 2003).

Jacob August FRANCKENSTEIN, Johann Conrad DINGLINGER, Diss. jur. de iuribus singularibus circa Judaeos, maxime in Germania (Leipzig 1722).

Sigmund Freud, Hemmung, Symptom und Angst (1926), in: Gesammelte Werke, Bd. XIV (Frankfurt am Main-London 1948) 111-205.

Winfried FreY, „Wölt Gott man hing sie wie die Hund". Vergleiche von Juden mit Hunden in deutschen Texten des Mittelalters und der frühen Neuzeit, in: Das Mittelalter. Perspektiven mediävistischer Forschung 12 (2007) 119-134.

Ludwig GeIGer (Hg.), Alexander VI. und sein Hof nach dem Tagebuch seines Zeremonienmeisters Burcardus (Stuttgart ${ }^{61913) . ~}$

David M. GITLITZ, Secrecy and Deceit. The Religion of the Crypto-Jews (Philadelphia-Jerusalem 1996).

Rudolf GlanZ, The 'Jewish Execution' in Medieval Germany, in: Jewish Social Studies 5 (1943) 3-26.

Christian Gottlieb GMELIN, Abhandlung von den besondern Rechten der Juden in peinlichen Sachen (Tübingen 1785).

Ludwig GÜNTHER, Idee der Wiedervergeltung in der Geschichte des deutschen Strafrechts (Gießen 1889).

Jacob GRIMM, Weisthümer, Bd. 1 (Göttingen 1840).

Hippolytus GUARINONIUS, Die Grewel der Verwüstung Menschlichen Geschlechts. In sieben vnter- 
schiedliche Bücher vnd vnmeidenliche Hauptstucken, sampt einem lustigen Vortrab abgetheilt [...] (Ingolstadt 1610)

Bernd HARDER, Das Lexikon der Großstadtmythen. Unglaubliche Geschichten von Astralreisen bis Zombies (Frankfurt am Main 2005).

Joel F. HARRINGTON, Die Ehre des Scharfrichters: Meister Frantz oder ein Henkersleben im 16. Jahrhundert. Aus dem Englischen von Norbert Juraschitz (München 2014).

Karl HÄRTER, Zur Stellung der Juden im frühneuzeitlichen Strafrecht, in: Andreas GotZMANN, Stephan WENDEHORST (Hgg.), Juden im Recht: neue Zugänge zur Rechtsgeschichte der Juden im Alten Reich (Berlin 2007) 347-379.

Clair HAYDEn BELL, Georg Hager. A Meistersinger of Nürnberg 1552-1634, 4 Bde. (Berkeley--os Angeles 1947).

Gerhard HEnschel, Neidgeschrei. Antisemitismus und Sexualität (Hamburg 2008).

Ludwig HerTel, Zum Wagnerkult im Nationalsozialismus. Ein Beitrag zur Rezeptionsgeschichte (Berlin 2015).

Magnus HirschFELD, Die Homosexualität des Mannes und des Weibes (Berlin 1914).

Historische Commission bei der königl. Academie der Wissenschaften (Hg.), Die Chroniken der fränkischen Städte, Nürnberg, Bd. 4 (Leipzig 1872).

Andreas HoNDORFF, Promptuarium exemplorum. [...] (Leipzig 1568).

Andreas HoNDORfF, Promptvarium exemplorum. [...](Frankfurt am Main 21572).

Joseph von HORMAYr, Anemonen aus dem Tagebuche eines alten Pilgersmannes, Bd. 1 (Jena 1845).

Nicole HorTZITZ, Die Sprache der Judenfeindschaft in der frühen Neuzeit 1450-1700. Untersuchungen zu Wortschatz, Text und Argumentation (Heidelberg 2005).

Hartwig von HUNDT-RADOWSKY, Judenspiegel. Ein Schand- und Sittengemälde alter und neuer Zeit, (Reutlingen 1821).

Le Jugement criminel rendu contre la synagogue des fripiers, portant que ceux de leur nombre qui se trouveront circoncis (qui est la marque de la Juifverie) seront chastrez ric à ric, afin que la race en demeure à jamais esteinte dans Paris (o. O. [1652]).

Judith KALIK, Fusion versus Alienation. Erotic Attraction, Sex, and Love between Jews and Christians in the Polish-Lithuanian Commonwealth, in: Yvonne KleINMANN (Hg.), Kommunikation durch symbolische Akte. Religiöse Heterogenität und politische Herrschaft in Polen-Litauen (Stuttgart 2010) 157-169.
Vera KALLENBERG, Der Streit um den ,Judenpurschen'. Interagierende Herrschafts- und Handlungsräume in der deutsch-jüdischen Geschichte HessenKassels und der Reichsritterschaft der Freiherrn von Thüngen um 1800. Ein Fallbeispiel, in: Stefan EHRENPREIS, Andreas GOTZMANN, Stephan WENDEHORST (Hgg.), Kaiser und Reich in der jüdischen Lokalgeschichte (München 2013) 93-115.

Akemi KANESHIRO-HAUPTMANN, „Das ist absolut wahr!" Wahre Geschichte oder moderne Sage? Rezeption der modernen Sagen im deutschsprachigen Raum (phil. Diss., Universität Göttingen 2010).

Debra KAPLAN, 'Because Our Wives Trade and Do Business with Our Goods'. Gender, Work, and Jewish-Christian Relations, in: Elisheva CARLEBACH, J. J. SCHACTER. (Hgg.), New Perspectives on Jewish-Christian Relations. In Honor of David Berger, (Leiden 2012) 241-261.

Cilli KASPER-HoltKotTE, Die jüdische Gemeinde von Frankfurt am Main in der Frühen Neuzeit. Familien Netzwerke und Konflikte eines jüdischen Zentrums (Berlin 2010).

Irena KuRZOVÀ, Priests in Dyer's Vat: Grotesque or Satire?, in: Neophilologus 97 (2013) 51-63.

Vito LA MANTIA, Roma e Stato romano (Rom 1884).

Jacques LACAN, Über die Bedeutung des Phallus. (1958), in: DERS., Schriften Bd. II, (BerlinWeinheim ${ }^{31991) ~ 121-132 . ~}$

Rodrigue LAVOIE, La délinquance sexuelle à Manosque (1240-1430). Schéma général et singularités juives, in: Provence historique 37 (1987) 371-387.

Gregorio LETI, Vita di Sisto Quinto pontefice romano, Bd. 3 (Turin 1852).

Israël LÉVI, L'Affaire Bourgeois, in: Revue des études juives 27 (1893) 180-206.

Jean LORIN, Commentarij in Librum Numeri [...], (Lyon 1622).

Karel MALÝ, Trestní právo v Čechách v 15.-16. Století (Praha/Prag 21989).

Giuseppe MAZZANTI, Habere rem cum iudea a christiano est rem habere cum cane iuris interpretatione. La damnata commixtio e il reato di bestialità nella dottrina giuridica di diritto commune, in: Historia et ius 11 (2017) 1-14.

Anca M. MIRON, Jack W. BREHM, Reactance Theory. 40 Years Later, in: Zeitschrift für Sozialpsychologie 37 (2006) 9-18.

Jörg R. MüLLER, "Sex and crime“ in Augsburg. Das Komplott gegen den Juden Joehlin im Jahre 1355, in: Frank G. HirschmanN, Gerd MentGen (Hgg.), Campana pulsante convocati. Festschrift zur Eme- 
ritierung von Prof. Dr. Alfred Haverkamp (Trier 2005) 395-419.

Jörg R. MÜLLER, Eine jüdische Diebesbande im Südwesten des Reiches in der ersten Hälfte des 14. Jahrhunderts, in: DERS. (Hg.), Beziehungsnetze aschkenasischer Juden während des Mittelalters und der frühen Neuzeit (Hannover 2008) 71-116.

DERS., Sexual Relationships between Christians and Jews in Medieval Germany, According to Christian Sources, in: Iggud. Selected Essays in Jewish Studies 2 (2005) 19-32.

Oldradus DE PONTE, Consilia, questiones \& allegatione (o.O. 1507).

Matthias POHLIG, Zwischen Gelehrsamkeit und konfessioneller Identitätsstiftung: lutherische Kirchenund Universalgeschichtsschreibung 1546-1617 (Tübingen 2007).

Paul RIEGER, Geschichte der Juden in Rom, Bd. 2: 1420-1870 (Berlin 1895).

Cecil RotH, Gleanings. Essays in Jewish history, letters and art (New York 1967).

Egon RUMBERG, Der außereheliche geschlechtliche Umgang mit Juden in rechtsgeschichtlicher Darstellung, in: Rasse und Recht 1 (1937/38) 397-407.

Emil Franz RösSLER, Die Stadtrechte von Brünn aus dem XIII. und XIV. Jahrhundert (Prag 1852).

Franco SACCHETTI, Die Novellen des Franco Sacchetti. Bürgers von Florenz, 3 Bde. (München 1907).

Car Anton SCHAAB, Diplomatische Geschichte der Juden in Mainz und dessen Umgebung (Mainz 1855).

Patrick SCHMIDT, Wandelbare Traditionen. Tradierter Wandel. Zünftische Erinnerungskulturen in der Frühen Neuzeit (Köln 2009).

Norbert SCHNITZLER, Juden vor Gericht. Soziale Ausrichtung durch Sanktionen, in: Hans SCHLOSSER, Rolf SPRANDEL, Dietmar WilloweIT (Hgg.), Herrschaftliches Strafen seit dem Hohen Mittelalter. Formen und Entwicklungsstufen (Köln 2002) 285308.

Jonathan SCHORSCH, Jews and Blacks in the Early Modern World (Cambridge 2004).

Peter SCHUSTER, Verbrecher, Opfer, Heilige. Eine Geschichte des Tötens 1200-1700 (Stuttgart 22016).

Johann Jacob ScHUDT, Jüdischer Merckwürdigkeiten IV. Theils (Frankfurt am Main 1717).

James SHAPIRO, Shakespeare and the Jews (New York 1996).

Joseph SHATZMILler, Jews, Medicine, and Medieval Society (Berkeley 1994).

Beatrice Silverman-WeINREICH, Towards a Structural Analysis of the Yiddish Proverb, in: Wolfgang
Mieder, Alan Dundes (Hgg.), The Wisdom of Many. Essays on the Proverb (London 1981) 6585.

Shlomo SimOnsoHn, History of the Jews in the Duchy of Mantua (Jerusalem 1977).

Pavla SlAvíČKOVÁ, Guardianship as a part of the Legal Protection of Children in Bohemia and Moravia before 1811, in: Geschichte und Region/Storia e regione 20 (2011) 106-125.

Irene STAHL, Die Meistersinger von Nürnberg (Nürnberg 1982) 179-184.

Leo STEINBERG, The Sexuality of Christ in Renaissance Art and in Modern Oblivion (Chicago 21996).

Sacha STERN, Jewish identity in early rabbinic writings (Leiden 1994).

Otto STовве, Die Juden in Deutschland während des Mittelalters (Braunschweig 1866).

Kenneth STOW, Jewish Dogs. An Image and Its Interpreters (Stanford 2006) XIV-XV.

[Jonathan SwIFT, James HoweLL], An Historical and law-treatise against Jews and Judaism [...] (London 1736).

Das Tagebuch des Meister Franz Scharfrichter zu Nürnberg. Nach der Buchausgabe von 1801. Kommentar von Jürgen Carl JACOBS und Heinz RÖLlEKE (Dortmund 1980).

Gary TAYLOR, Castration. An Abbreviated History of Western Manhood (London 2000).

Magda Teter, Jews and Heretics in Catholic Poland. A Beleaguered Church in the Post-Reformation Era (Cambridge 2006).

Ariel TOAFF, Love, Work, and Death. Jewish Life in Medieval Umbria (London 1998).

Susan TUCHEL, Kastration im Mittelalter (Düsseldorf 1998).

Chiara VAlseCCHI, Oldrado da Ponte e i suoi consilia. Un' auctoritas del primo trecento (Mailand 2000).

Klaus VAN EICKELS, Gendered Violence: Castration and Blinding as Punishment for Treason in Normandy and Anglo-Norman England, in: Gender \& History 16 (2004) 588-602.

Martina Vordermayer, Antisemitismus und Judentum bei Clemens Brentano (Frankfurt am Main 1999).

Johann Christoph WAGENSEIL: Locvlamentvm Tertivm; [Pars 1]: Insunt: Adminicula Studii Historici. Scilicet: Mythologia Veterum. Praecognita Historica. Mnemonica Historica. Synopsis Historiae Vniversalis. Nomenclator Regum, \& Principum Europa. (Nürnberg 1695). 
Roni WeINSTEIN, Marriage Rituals Italian Style. A Historical Anthropological Perspective on Early Modern Italian Jews (Leiden 2004).

DERS., Juvenile sexuality, Kabbalah and Catholic reformation in Italy. Tiferet Bahurim by Pinhas Barukh ben Pelatiyah Monselice (Leiden 2009).

Christian WiLdvogel, Johann Carl Lochner, Dodecas legalis de Iudaeorum receptione ac tolerantia. Von Juden-Schutz [...], (Jena 1730).

Christlieb WuchERFEIND [Pseudonym], Der verdammliche Juden-Spieß, oder rechtmäßiges und historisches Bedencken von der Juden verdammlicher Geldwucher [...] (o. O. 1688).
Norman ZACOUR, Jews and Saracens in the Consilia of Oldradus de Ponte (Toronto 1990).

Helmut ZeDELMAIER, Navigieren im Text-Universum. Theodor Zwingers Theatrum Vitae Humanae, in: metaphorik.de 14 (2008) 113-135.

Natalie Zemon Davis, Fiction in the Archives. Pardon Tales and Their Tellers in Sixteenth-century France (Stanford 1997).

Theodor ZWINGER, Theatrum vitae humanae [...] (Basel 1565, 21571).

Volumen statutorum civitatis Maceratae (o. O. 1553). 